\title{
GUIDED CIRCUMFERENTIAL WAVES IN LAYERED POROELASTIC CYLINDERS
}

\author{
S.A. SHAH ${ }^{*}$ \\ Department of Mathematics \\ Deccan College of Engineering and Technology \\ Hyderabad-500 001 (T.S) INDIA \\ E-mail: ahmed_shah67@yahoo.com \\ G. APSAR \\ Department of Mechanical Engineering \\ Deccan College of Engineering and Technology \\ Hyderabad-500 001 (T.S), INDIA
}

\begin{abstract}
The present paper investigates the propagation of time harmonic circumferential waves in a two-dimensional hollow poroelastic cylinder with an inner shaft (shaft-bearing assembly). The hollow poroelastic cylinder and inner shaft are assumed to be infinite in axial direction. The outer surface of the cylinder is stress free and at the interface, between the inner shaft and the outer cylinder, it is assumed to be free sliding and the interfacial shear stresses are zero, also the normal stress and radial displacements are continuous. The frequency equation of guided circumferential waves for a permeable and an impermeable surface is obtained. When the angular wave number vanish the frequency equation of guided circumferential waves for a permeable and an impermeable surface degenerates and the dilatational and shear waves are uncoupled. Shear waves are independent of the nature of surface. The frequency equation of a permeable and an impermeable surface for bore-piston assembly is obtained as a particular case of the model under consideration when the outer radius of the hollow poroelastic cylinder tends to infinity. Results of previous studies are obtained as a particular case of the present study. Nondimensional frequency as a function of wave number is presented graphically for two types of models and discussed. Numerical results show that, in general, the first modes are linear for permeable and impermeable surfaces and the frequency of a permeable surface is more than that of an impermeable surface.
\end{abstract}

Key words: Biot's theory, poroelastic layered cylinder, circumferential waves, shaft-bearing assembly, borepiston assembly, permeable surface, impermeable surface, wave number, frequency.

\section{Introduction}

Most of components in engineering usually consist of cylindrical structures composed of at least an inner cylinder and an outer cylinder. Study of fatigue cracks in cylindrical components is important, because due to the application of a load, crack formation follows. These fatigue cracks generally form at the interface between the two layers and they spread in a radial direction. Detection of such radial cracks is important to avoid a severe mechanical failure. A guided wave propagates in the direction of the layer, behaving as a standing wave through the thickness of the layer. Guided waves are commonly used to examine simple structures such as beams and plates and layered cylinders. One such example is given by Nagy et al [1], who suggested that radial fatigue cracks in weep holes in airframes may be detected by using guided waves propagating along the circumferential direction of the hole. Christine Valle et al [2] investigated the guided circumferential waves in layered cylinders and showed that the waves are non dispersive at high frequency or high wave number. Tajuddin and Shah $[3,4]$ studied the circumferential waves and torsional vibrations of

\footnotetext{
* To whom correspondence should be addressed
} 
hollow poroelastic cylinders in the presence of dissipation and found that the phase velocity is non-dispersive in the case of circumferential waves. Whittier and Jones [5] studied axially symmetric vibrations in a twolayered cylinder. Guided circumferential waves in a circular annulus are studied by Liu and Qu [6]. Malla Reddy and Tajuddin [7] studied the plane-strain vibrations of thick-walled hollow poroelastic cylinders. Towfighi et al. [8] discussed the wave propagation in a circumferential direction in anisotropic cylindrical curved plates. Shah [9] presented the solutions for flexural wave propagation in coated poroelastic cylinders where the rule of mixtures is used to understand the dispersion phenomenon in a coated cylinder knowing the frequency equations for the cases of homogeneous poroelastic cylinders made of coating and core poroelastic materials separately.

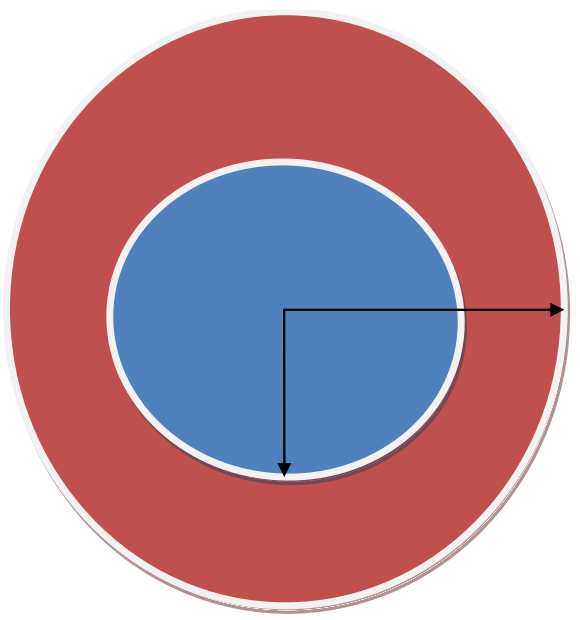

Fig.1. Cross-section of shaft-bearing assembly.

In this paper, guided circumferential waves are studied for the purpose of detecting the radial fatigue cracks in a shaft-bearing assembly that is, a layered poroelastic cylinder. The frequency equation obtained helps in understanding the radial fatigue cracks. For this purpose, it is necessary to understand the behavior of wave propagation in a layered poroelastic cylinder. Time harmonic guided wave propagation in a circumferential direction of a double layered poroelastic cylinder (consisting of a shaft-bearing assembly) is considered. The poroelastic materials of each shaft and bearing are assumed to be homogeneous and isotropic. The boundary condition at the interface [between the inner shaft and the bearing (outer hollow cylinder)] is assumed to be free-sliding; that is, the interfacial shear stresses are assumed to be zero, and the normal stress and radial displacements are continuous. The frequency equations are obtained for the model under consideration by using Biot's [10] theory of wave propagation. The frequency equation of a permeable and an impermeable surface for the cylindrical-piston assembly is obtained as a particular case of the model under consideration when the outer radius of the hollow poroelastic cylinder tends to infinity. When the angular wave number vanishes, the dilatational and shear waves are uncoupled in both the models and the frequency equation of shear waves is independent of the nature of the surface, that is, it is same for permeable and impermeable surfaces. Numerical results for the dispersion curves of the first three modes are presented and discussed.

\section{Governing equations}

The equations of motion of a homogeneous, isotropic poroelastic solid (Biot [10]) in the presence of dissipation $b$ are 


$$
\begin{aligned}
& N \nabla^{2} \boldsymbol{u}+(A+N) \nabla e+Q \nabla \in=\frac{\partial^{2}}{\partial t^{2}}\left(\rho_{11} \boldsymbol{u}+\rho_{12} \boldsymbol{U}\right)+b \frac{\partial}{\partial t}(\boldsymbol{u}-\boldsymbol{U}), \\
& Q \nabla e+R \nabla \in=\frac{\partial^{2}}{\partial t^{2}}\left(\rho_{12} \boldsymbol{u}+\rho_{22} \boldsymbol{U}\right)-b \frac{\partial}{\partial t}(\boldsymbol{u}-\boldsymbol{U})
\end{aligned}
$$

where $\nabla^{2}$ is the Laplacian, $\boldsymbol{u}\left(u_{r}, u_{\theta}, u_{z}\right)$ and $\boldsymbol{U}\left(U_{r}, U_{\theta}, U_{z}\right)$ are displacements of the solid and liquid respectively, $e$ and $\in$ are the dilatations of solid and liquid; $A, N, Q, R$ are poroelastic constants and $\rho_{j k}(j, k=$ $1,2)$ are the mass coefficients following Biot [10]. The poroelastic constants $A, N$ correspond to familiar Lame constants in a purely elastic solid. The coefficient $N$ represents the shear modulus of the solid. The coefficient $\mathrm{R}$ is a measure of the pressure required on the liquid to force a certain amount of the liquid into the aggregate while the total volume remains constant. The coefficient $Q$ represents the coupling between the volume change of the solid to that of the liquid.

For the present problem, we consider the displacement components of solid $\boldsymbol{u}\left(u_{r}, u_{\theta}, 0\right)$ and liquid $\boldsymbol{U}\left(U_{r}, U_{\theta}, 0\right)$

$$
\begin{array}{ll}
u_{r}=\frac{\partial \Phi_{1}}{\partial r}+\frac{1}{r} \frac{\partial \Psi_{1}}{\partial \theta}, & u_{\theta}=\frac{1}{r} \frac{\partial \Phi_{1}}{\partial \theta}-\frac{\partial \Psi_{1}}{\partial r}, \\
U_{r}=\frac{\partial \Phi_{2}}{\partial r}+\frac{1}{r} \frac{\partial \Psi_{2}}{\partial \theta}, & U_{\theta}=\frac{1}{r} \frac{\partial \Phi_{2}}{\partial \theta}-\frac{\partial \Psi_{2}}{\partial r}
\end{array}
$$

where $\Phi_{1}, \Phi_{2}, \psi_{1}, \psi_{2}$ are functions of $r, \theta$ and time $t$.

Substitution of Eq.(2.2) into Eq.(2.1) yields

$$
\begin{aligned}
& P \nabla^{2} \Phi_{1}+Q \nabla^{2} \Phi_{2}=\frac{\partial^{2}}{\partial t^{2}}\left(\rho_{11} \Phi_{1}+\rho_{12} \Phi_{2}\right)+b \frac{\partial}{\partial t}\left(\Phi_{1}-\Phi_{2}\right), \\
& Q \nabla^{2} \Phi_{1}+R \nabla^{2} \Phi_{2}=\frac{\partial^{2}}{\partial t^{2}}\left(\rho_{12} \Phi_{1}+\rho_{22} \Phi_{2}\right)-b \frac{\partial}{\partial t}\left(\Phi_{1}-\Phi_{2}\right), \\
& N \nabla^{2} \Psi_{1}=\frac{\partial^{2}}{\partial t^{2}}\left(\rho_{11} \Psi_{1}+\rho_{12} \Psi_{2}\right)+b \frac{\partial}{\partial t}\left(\Psi_{1}-\Psi_{2}\right), \\
& 0=\frac{\partial^{2}}{\partial t^{2}}\left(\rho_{12} \Psi_{1}+\rho_{22} \Psi_{2}\right)-b \frac{\partial}{\partial t}\left(\Psi_{1}-\Psi_{2}\right)
\end{aligned}
$$

where $P=A+2 N$ and $\nabla^{2}=\frac{\partial^{2}}{\partial r^{2}}+\frac{1}{r} \frac{\partial}{\partial r}+\frac{1}{r^{2}} \frac{\partial^{2}}{\partial \theta^{2}}$.

The stresses $\sigma_{j k}$ and the liquid pressure $s$ of the poroelastic solid are

$$
\begin{aligned}
& \sigma_{j k}=2 N e_{j k}+(A e+Q \in) \delta_{j k}, \quad(j, k=r, \theta, z), \\
& s=Q e+R \in
\end{aligned}
$$

where $\delta_{j k}$ is the well-known Kronecker delta function. 


\section{Solution of the problem}

We consider a two layered poroelastic cylinder of infinite extent in the axial direction whose cross section is shown in Fig.1, where the axis of the cylinder is along the $z$-axis and $(r, \theta, z)$ are the cylindrical polar co-ordinates. Each layer of the poroelastic cylinder is isotropic and homogeneous. The radius of the inner shaft is $r_{1}$ and outer radius of the bearing (hollow cylinder) is $r_{2}$. For waves propagating in the circumferential direction the assumption of plane-strain deformation prevails. In this case, the pertinent nonzero displacement and stress components are $u_{r}, u_{\theta}, \sigma_{r r}, \sigma_{\theta \theta}, \sigma_{r \theta}$ and these are the variables of $r$ and $\theta$ only. The physical parameters related to the inner shaft are denoted by * as a superscript. For example, poroelastic constants of the poroelastic outer cylinder (bearing) are $A, N, Q, R$ and the poroelastic constants of the inner shaft are denoted by $A^{*}, N^{*}, Q^{*}, R^{*}$. We seek the solution of Eqs $(2.3)$ in the form

$$
\begin{array}{ll}
\Phi_{1}=F_{1}(r) e^{i(n \theta-\omega t)}, & \Phi_{2}=F_{2}(r) e^{i(n \theta-\omega t)}, \\
\Psi_{1}=G_{1}(r) e^{i(n \theta-\omega t)}, & \Psi_{2}=G_{2}(r) e^{i(n \theta-\omega t)}
\end{array}
$$

where $n$ is the angular wave number $\omega$ is the circular frequency and $i$ is the complex unity. Equation (3.1) represents the potentials functions for time harmonic guided waves propagating in the circumferential direction and it is a model proposed by Viktorov [11] and then subsequently used by other authors. By substituting Eqs (3.1) into Eqs (2.3), its solution (bounded solution for the inner shaft) yields

$$
\begin{aligned}
& F_{1}= \begin{cases}{\left[C_{1} J_{n}\left(\xi_{1} r\right)+C_{2} Y_{n}\left(\xi_{1} r\right)+C_{3} J_{n}\left(\xi_{2} r\right)+C_{4} Y_{n}\left(\xi_{2} r\right)\right],} & r_{1}<r<r_{2}, \\
{\left[D_{1} J_{n}\left(\xi_{1} r\right)+D_{2} J_{n}\left(\xi_{2} r\right)\right],} & 0<r<r_{1},\end{cases} \\
& F_{2}= \begin{cases}-\left[C_{1} \delta_{1}^{2} J_{n}\left(\xi_{1} r\right)+C_{2} \delta_{1}^{2} Y_{n}\left(\xi_{1} r\right)+C_{3} \delta_{2}^{2} J_{n}\left(\xi_{2} r\right)+C_{4} \delta_{2}^{2} Y_{n}\left(\xi_{2} r\right)\right], & r_{1}<r<r_{2}, \\
-\left[D_{1} \delta_{1}^{2} J_{n}\left(\xi_{1} r\right)+D_{2} \delta_{2}^{2} J_{n}\left(\xi_{2} r\right)\right], & 0<r<r_{1},\end{cases} \\
& G_{1}= \begin{cases}{\left[C_{5} J_{n}\left(\xi_{3} r\right)+C_{6} Y_{n}\left(\xi_{3} r\right)\right],} & r_{1}<r<r_{2}, \\
{\left[D_{3} J_{n}\left(\xi_{3} r\right)\right],} & 0<r<r_{1},\end{cases} \\
& G_{2}=-K_{12} K_{22}^{-1} \begin{cases}{\left[C_{5} J_{n}\left(\xi_{3} r\right)+C_{6} Y_{n}\left(\xi_{3} r\right)\right],} & r_{1}<r<r_{2}, \\
{\left[D_{3} J_{n}\left(\xi_{3} r\right)\right],} & 0<r<r_{1} .\end{cases}
\end{aligned}
$$

In Eqs (3.2), (3.3), (3.4), (3.5), $C_{1}, C_{2}, C_{3}, C_{4}, C_{5}, C_{6}, D_{1}, D_{2}, D_{3}$ are arbitrary constants, $J_{n}$ and $Y_{n}$ are Bessel functions of first and second kind each of order $n$ and

$$
\xi_{j}=\frac{\omega}{V_{j}}, \quad(j=1,2,3), \quad \delta_{j}^{2}=\frac{\left[\left(R K_{11}-Q K_{12}\right)-V_{j}^{-2}\left(P R-Q^{2}\right)\right]}{\left(R K_{12}-Q K_{22}\right)}, \quad(j=1,2) .
$$


In Eqs (3.6), $V_{1}$ and $V_{2}$ are the velocities of dilatational waves of first and second kind respectively, $V_{3}$ is the shear wave velocity and

$$
K_{11}=\rho_{11}-\frac{i b}{\omega}, \quad K_{12}=\rho_{12}+\frac{i b}{\omega}, \quad K_{22}=\rho_{22}-\frac{i b}{\omega} .
$$

Employing Eqs (3.2), (3.3), (3.4), (3.5) into Eqs (3.1) and then using Eqs (2.2) we get the displacement components of the solid as

$$
\begin{aligned}
& u_{r}=\left\{\begin{array}{l}
\left\{\left[C_{1} \xi_{1} J_{n}^{\prime}\left(\xi_{1} r\right)+C_{2} \xi_{1} Y_{n}^{\prime}\left(\xi_{1} r\right)+C_{3} \xi_{2} J_{n}^{\prime}\left(\xi_{2} r\right)+C_{4} \xi_{2} Y_{n}^{\prime}\left(\xi_{2} r\right)\right]+\right. \\
\left.+\frac{i n}{r}\left[C_{5} J_{n}\left(\xi_{3} r\right)+C_{6} Y_{n}\left(\xi_{3} r\right)\right]\right\} e^{i(n \theta-\omega t)}, \quad r_{1}<r<r_{2}, \\
\left\{D_{1} \xi_{1}^{*} J_{n}^{\prime}\left(\xi_{1}^{*} r\right)+D_{2} \xi_{2}^{*} J_{n}^{\prime}\left(\xi_{2}^{*} r\right)+D_{3} \frac{i n}{r} J_{n}\left(\xi_{3}^{*} r\right)\right\} e^{i(n \theta-\omega t)}, \quad 0<r<r_{1},
\end{array}\right. \\
& u_{\theta}= \begin{cases}\left\{\frac{i n}{r}\left[C_{1} J_{n}\left(\xi_{1} r\right)+C_{2} Y_{n}\left(\xi_{1} r\right)+C_{3} J_{n}\left(\xi_{2} r\right)+C_{4} Y_{n}\left(\xi_{2} r\right)\right]+\right. \\
\left.-\xi_{3}\left[C_{5} J_{n}^{\prime}\left(\xi_{3} r\right)+C_{6} \xi_{3} Y_{n}^{\prime}\left(\xi_{3} r\right)\right]\right\} e^{i(n \theta-\omega t)}, \quad r_{1}<r<r_{2}, \\
\left\{\frac{i n}{r}\left[D_{1} J_{n}\left(\xi_{1}^{*} r\right)+D_{2} J_{n}\left(\xi_{2}^{*} r\right)\right]-D_{3} \xi_{3}^{*} J_{n}^{\prime}\left(\xi_{3}^{*} r\right)\right\} e^{i(n \theta-\omega t),}, 0<r<r_{1},\end{cases}
\end{aligned}
$$

where a dash (/) over a quantity represents the differentiation with respect to $r$.

The non-zero stresses and liquid pressure in terms of potential functions are

$$
\begin{aligned}
\sigma_{r r} & =2 N\left(\frac{\partial^{2} \Phi_{1}}{\partial r^{2}}-\frac{1}{r^{2}} \frac{\partial \Psi_{1}}{\partial \theta}+\frac{1}{r} \frac{\partial^{2} \Psi_{1}}{\partial \theta \partial r}\right)+A \nabla^{2} \Phi_{1}+Q \nabla^{2} \Phi_{2}, \\
\sigma_{r \theta} & =N\left(\frac{2}{r} \frac{\partial^{2} \Phi_{1}}{\partial r \partial \theta}-\frac{2}{r^{2}} \frac{\partial \Phi_{1}}{\partial \theta}-\frac{\partial^{2} \Psi_{1}}{\partial r^{2}}+\frac{1}{r^{2}} \frac{\partial^{2} \Psi_{1}}{\partial \theta^{2}}+\frac{1}{r} \frac{\partial \Psi_{1}}{\partial r}\right), \\
S & =Q \nabla^{2} \Phi_{1}+R \nabla^{2} \Phi_{2} \\
\text { where } \quad \nabla^{2} & =\frac{\partial^{2}}{\partial r^{2}}+\frac{1}{r} \frac{\partial}{\partial r}+\frac{1}{r^{2}} \frac{\partial^{2}}{\partial \theta^{2}} .
\end{aligned}
$$

\section{Boundary conditions - frequency equation}

The outer surface of the cylinder (bearing) is assumed to be stress free and at the interface, the inner shaft and the outer cylinder (bearing) is free-sliding. Thus the boundary conditions for free vibrations in the case of a permeable surface are 


$$
\begin{array}{lcc}
\left(\sigma_{r r}+s\right)_{r=r_{I}^{+}}-\left(\sigma_{r r}^{*}+s^{*}\right)_{r=r_{I}^{-}}=0, & \left(\sigma_{r \theta}\right)_{r=r_{I}^{+}}=0, & \left(\sigma_{r \theta}^{*}\right)_{r=r_{I}^{-}}=0, \\
\left(u_{r}\right)_{r=r_{I}^{+}}-\left(u_{r}^{*}\right)_{r=r_{I}^{-}}=0, & (s)_{r=r_{I}^{+}}=0, & \left(s^{*}\right)_{r=r_{I}^{-}}=0, \\
\sigma_{r r}+s=0, \quad \sigma_{r \theta}=0, & s=0, \quad \text { at } \quad r=r_{2} .
\end{array}
$$

Similarly, the boundary conditions in the case of an impermeable surface are

$$
\begin{array}{lll}
\left(\sigma_{r r}+s\right)_{r=r_{I}^{+}}-\left(\sigma_{r r}^{*}+s^{*}\right)_{r=r_{I}^{-}}=0, & \left(\sigma_{r \theta}\right)_{r=r_{I}^{+}}=0, & \left(\sigma_{r \theta}^{*}\right)_{r=r_{I}^{-}}=0, \\
\left(u_{r}\right)_{r=r_{I}^{+}}-\left(u_{r}^{*}\right)_{r=r_{I}^{-}}=0, & \left.\left(\frac{\partial s}{\partial r}\right)_{r=r_{I}^{+}}^{*}\right)_{r=r_{I}^{-}}=0, & \\
\sigma_{r r}+s=0, \quad \sigma_{r \theta}=0, & \frac{\partial s}{\partial r}=0, \quad \text { at } \quad r=r_{2} .
\end{array}
$$

Putting Eqs (2.4), (3.8) and (3.9) into Eqs (4.1) results in a system of nine homogeneous algebraic equations of nine constants $C_{1}, C_{2}, C_{3}, C_{4}, C_{5}, C_{6}, D_{1}, D_{2}$, and $D_{3}$. For a non-trivial solution to exist, the determinant of the coefficients must vanish. By eliminating these constants, the frequency equation of guided circumferential waves of a layered poroelastic cylinder in the case of a permeable surface is

$$
\left|M_{j k}\right|=0, \quad j, k=1,2,3 \ldots 9
$$

where the elements $M_{j k}(r)$ are given in the Appendix [see Eq.(A.1)].

Arguing similarly, putting Eqs (2.4), (3.8) and (3.9) into Eqs (4.2) yields the frequency equation of guided circumferential waves of a layered poroelastic cylinder in the case of an impermeable surface to be

$$
\left|N_{j k}\right|=0, \quad j, k=1,2,3 \ldots 9
$$

where the elements $N_{j k}(r)$ are given in the Appendix [see Eq.(A.2)].

By eliminating liquid effects from the frequency equation of a permeable surface (4.3), that is, setting $b \rightarrow 0, \rho_{12} \rightarrow 0, \rho_{22} \rightarrow 0,\left(A-Q^{2} / R\right) \rightarrow \lambda, N \rightarrow \mu, Q \rightarrow 0, R \rightarrow 0$ and after rearrangement of the terms, the results for a purely elastic solid are obtained as a particular case considered by Christine Valle et al. [2]. The frequency equation of an impermeable surface (4.4) has no counterpart in the purely elastic solid.

Now we consider two particular cases of the general frequency Eqs (4.3) and (4.4) of guided circumferential waves: (i) When the angular wave number vanishes. (ii) When the outer radius of the hollow poroelastic cylinder tends to infinity, the model under consideration (shaft-bearing assembly) reduces to the cylinder-piston assembly.

(i) When the angular wave number $\mathrm{n}$ vanish, the frequency equation of a permeable surface that is Eqs (4.3) degenerates into the product

$$
M_{39}\left(r_{1}\right) D_{1} D_{2}=0
$$

where $M_{j k}(r)$ are defined in the Appendix [see Eq.(A.1)] and $D_{1}, D_{2}$ are 


$$
\begin{aligned}
D_{1} & =\left|\begin{array}{lllll}
M_{25}\left(r_{1}\right) & M_{26}\left(r_{1}\right) \\
M_{85}\left(r_{2}\right) & M_{85}\left(r_{2}\right)
\end{array}\right|, \\
D_{2} & =\left|\begin{array}{cccccc}
M_{11}\left(r_{1}\right) & M_{12}\left(r_{1}\right) & M_{13}\left(r_{1}\right) & M_{14}\left(r_{1}\right) & M_{17}\left(r_{1}\right) & M_{18}\left(r_{1}\right) \\
M_{41}\left(r_{1}\right) & M_{42}\left(r_{1}\right) & M_{43}\left(r_{1}\right) & M_{44}\left(r_{1}\right) & M_{47}\left(r_{1}\right) & M_{48}\left(r_{1}\right) \\
M_{51}\left(r_{1}\right) & M_{52}\left(r_{1}\right) & M_{53}\left(r_{1}\right) & M_{54}\left(r_{1}\right) & 0 & 0 \\
0 & 0 & 0 & 0 & M_{67}\left(r_{1}\right) & M_{68}\left(r_{1}\right) \\
M_{71}\left(r_{2}\right) & M_{72}\left(r_{2}\right) & M_{73}\left(r_{2}\right) & M_{74}\left(r_{2}\right) & 0 & 0 \\
M_{91}\left(r_{2}\right) & M_{92}\left(r_{2}\right) & M_{93}\left(r_{2}\right) & M_{94}\left(r_{2}\right) & 0 & 0
\end{array}\right| .
\end{aligned}
$$

Similarly, when the wave number $\mathrm{n}$ vanishes, the frequency equation of an impermeable surface [Eq.(4.4)] degenerates into the product

$$
N_{39}\left(r_{1}\right) D_{3} D_{4}=0
$$

where $N_{j k}(r)$ are defined in the appendix [see Eq.(A.2)] and $D_{3}, D_{4}$ are

$$
\begin{aligned}
D_{3} & =\left|\begin{array}{ll}
N_{25}\left(r_{1}\right) & N_{26}\left(r_{1}\right) \\
N_{85}\left(r_{2}\right) & N_{85}\left(r_{2}\right)
\end{array}\right|, \\
D_{4} & =\left|\begin{array}{cccccc}
N_{11}\left(r_{1}\right) & N_{12}\left(r_{1}\right) & N_{13}\left(r_{1}\right) & N_{14}\left(r_{1}\right) & N_{17}\left(r_{1}\right) & N_{18}\left(r_{1}\right) \\
N_{41}\left(r_{1}\right) & N_{42}\left(r_{1}\right) & N_{43}\left(r_{1}\right) & N_{44}\left(r_{1}\right) & N_{47}\left(r_{1}\right) & N_{48}\left(r_{1}\right) \\
N_{51}\left(r_{1}\right) & N_{52}\left(r_{1}\right) & N_{53}\left(r_{1}\right) & N_{54}\left(r_{1}\right) & 0 & 0 \\
0 & 0 & 0 & 0 & N_{67}\left(r_{1}\right) & N_{68}\left(r_{1}\right) \\
N_{71}\left(r_{2}\right) & N_{72}\left(r_{2}\right) & N_{73}\left(r_{2}\right) & N_{74}\left(r_{2}\right) & 0 & 0 \\
N_{91}\left(r_{2}\right) & N_{92}\left(r_{2}\right) & N_{93}\left(r_{2}\right) & N_{94}\left(r_{2}\right) & 0 & 0
\end{array}\right| .
\end{aligned}
$$

Equation (4.5) is satisfied if, $M_{39}\left(r_{1}\right)=0$, or $D_{1}=0$ or $D_{2}=0$. Equation $M_{39}\left(r_{1}\right)=0$, when simplified reduces to $J_{2}\left(\xi_{3}^{*} r_{1}\right)=0$, which is similar to the frequency equation of torsional vibrations discussed by Tajuddin and Sarma [12]. The equation $D_{1}=0$, when simplified reduces to $J_{2}\left(\xi_{3} r_{1}\right) Y_{2}\left(\xi_{3} r_{2}\right)-J_{2}\left(\xi_{3} r_{2}\right) Y_{2}\left(\xi_{3} r_{1}\right)=0$ which is the frequency equation of axially symmetric shear vibrations discussed by Malla Reddy and Tajuddin [7] and also similar to the frequency equation of torsional vibrations of hollow poroelastic cylinders discussed by Tajuddin and Ahmed Shah [4]. The equation

$$
D_{2}=0
$$

is the frequency equation of dilatational guided circumferential waves of the shaft-bearing assembly in the case of a permeable surface. From Eq.(4.5) we see that the dilatational and shear waves are uncoupled. It is important to note that the shear waves are uncoupled in the outer poroelastic cylinder (bearing) and the inner shaft. But the dilatational waves are coupled in shaft-bearing assembly. As discussed in the case of a permeable surface, from Eq.(4.7) we see that the frequency equation of dilatational circumferential waves for an impermeable surface is

$$
D_{4}=0 \text {, }
$$


also it can be noted that the frequency equation of shear vibrations is independent of nature of surface, that is, it is same for a permeable and an impermeable surface.

(ii) When the outer radius of the outer poroelastic hollow cylinder (bearing) tends to infinity, the shaftbearing model reduces to the cylinder-piston assembly. Analogous displacements and stresses for a circular cylindrical cavity in an infinite porous medium are written replacing Bessel functions of first and second kind by Bessel function of third kind (Hankel function), $H_{n}^{\left({ }^{(I)}\right.}$ and $H_{n}{ }^{(2)}$. Hankel functions may be expressed in the form of Bessel functions of first and second kind

$$
H_{n}^{(1)}=J_{n}+i Y_{n}, \quad H_{n}^{(2)}=J_{n}-i Y_{n} .
$$

The Hankel function tends to zero as the argument becomes large, although the individual functions do not. For all values of $n$, the Hankel functions $H_{n}^{\left({ }^{(1)}\right.}$ and $H_{n}^{(2)}$ are linearly independent. $H_{n}^{\left({ }^{(1)}\right.}$ represents waves propagating towards the origin while $H_{n}^{(2)}$ represents waves diverging from the origin. Since there is no outer boundary, the terms containing $H_{n}{ }^{(I)}$ must vanish. The displacement and stresses may then be written in terms of $H_{n}^{(2)}$. We see that after necessary calculations, $H_{n}^{(2)}$ is to be transformed to the Bessel function of second kind $Y_{n}$. Then the frequency equation of guided circumferential waves for the cylinderpiston assembly in the case of a permeable surface is

$$
\left|\begin{array}{cccccc}
M_{12}\left(r_{1}\right) & M_{14}\left(r_{1}\right) & M_{16}\left(r_{1}\right) & M_{17}\left(r_{1}\right) & M_{18}\left(r_{1}\right) & M_{19}\left(r_{1}\right) \\
M_{22}\left(r_{1}\right) & M_{24}\left(r_{1}\right) & M_{26}\left(r_{1}\right) & 0 & 0 & 0 \\
0 & 0 & 0 & M_{37}\left(r_{1}\right) & M_{38}\left(r_{1}\right) & M_{39}\left(r_{1}\right) \\
M_{42}\left(r_{1}\right) & M_{44}\left(r_{1}\right) & M_{46}\left(r_{1}\right) & M_{47}\left(r_{1}\right) & M_{48}\left(r_{1}\right) & M_{49}\left(r_{1}\right) \\
M_{52}\left(r_{1}\right) & M_{54}\left(r_{1}\right) & 0 & 0 & 0 & 0 \\
0 & 0 & 0 & M_{67}\left(r_{1}\right) & M_{68}\left(r_{1}\right) & 0
\end{array}\right|=0 .
$$

Similarly, the frequency equation of guided circumferential waves for the cylinder-piston assembly in the case of an impermeable surface is

$$
\left|\begin{array}{cccccc}
N_{12}\left(r_{1}\right) & N_{14}\left(r_{1}\right) & N_{16}\left(r_{1}\right) & N_{17}\left(r_{1}\right) & N_{18}\left(r_{1}\right) & N_{19}\left(r_{1}\right) \\
N_{22}\left(r_{1}\right) & N_{24}\left(r_{1}\right) & N_{26}\left(r_{1}\right) & 0 & 0 & 0 \\
0 & 0 & 0 & N_{37}\left(r_{1}\right) & N_{38}\left(r_{1}\right) & N_{39}\left(r_{1}\right) \\
N_{42}\left(r_{1}\right) & N_{44}\left(r_{1}\right) & N_{46}\left(r_{1}\right) & N_{47}\left(r_{1}\right) & N_{48}\left(r_{1}\right) & N_{49}\left(r_{1}\right) \\
N_{52}\left(r_{1}\right) & N_{54}\left(r_{1}\right) & 0 & 0 & 0 & 0 \\
0 & 0 & 0 & N_{67}\left(r_{1}\right) & N_{68}\left(r_{1}\right) & 0
\end{array}\right|=0 .
$$

In Eqs (4.11) and (4.12), the elements $M_{j k}(r)$ and $N_{j k}(r)$ are defined in Eqs (A.1) and (A.2), respectively, in the appendix. Frequency Eqs (4.11) and (4.12) can be considered for zero angular wave number.

For further analysis of frequency Eqs (4.3) and (4.4), now we denote these equations by $\Delta_{1}$ and $\Delta_{2}$, respectively. After necessary simplifications, Eq.(4.3) can be re-written as

$$
\left|\Delta_{1}\right|=\left|\begin{array}{ll}
\Delta_{11} & \Delta_{12} \\
\Delta_{21} & \Delta_{22}
\end{array}\right|=0
$$

where the sub-determinants $\Delta_{j k}(j, k=1,2)$ are 


$$
\begin{aligned}
& \Delta_{11}=\left|\begin{array}{ccc}
M_{37}\left(r_{1}\right) & M_{38}\left(r_{1}\right) & M_{39}\left(r_{1}\right) \\
M_{47}\left(r_{1}\right) & M_{48}\left(r_{1}\right) & M_{49}\left(r_{1}\right) \\
M_{67}\left(r_{1}\right) & M_{68}\left(r_{1}\right) & 0
\end{array}\right|, \quad \Delta_{12}=\left|\begin{array}{ccc}
M_{17}\left(r_{1}\right) & M_{18}\left(r_{1}\right) & M_{19}\left(r_{1}\right) \\
M_{37}\left(r_{1}\right) & M_{38}\left(r_{1}\right) & M_{39}\left(r_{1}\right) \\
M_{67}\left(r_{1}\right) & M_{68}\left(r_{1}\right) & 0
\end{array}\right|, \\
& \Delta_{21}=\left|\begin{array}{llllcc}
M_{21}\left(r_{1}\right) & M_{22}\left(r_{1}\right) & M_{23}\left(r_{1}\right) & M_{24}\left(r_{1}\right) & M_{25}\left(r_{1}\right) & M_{26}\left(r_{1}\right) \\
M_{41}\left(r_{1}\right) & M_{42}\left(r_{1}\right) & M_{43}\left(r_{1}\right) & M_{44}\left(r_{1}\right) & M_{45}\left(r_{1}\right) & M_{46}\left(r_{1}\right) \\
M_{51}\left(r_{1}\right) & M_{52}\left(r_{1}\right) & M_{53}\left(r_{1}\right) & M_{54}\left(r_{1}\right) & 0 & 0 \\
M_{71}\left(r_{2}\right) & M_{72}\left(r_{2}\right) & M_{73}\left(r_{2}\right) & M_{74}\left(r_{2}\right) & M_{75}\left(r_{2}\right) & M_{76}\left(r_{2}\right) \\
M_{81}\left(r_{2}\right) & M_{82}\left(r_{2}\right) & M_{83}\left(r_{2}\right) & M_{84}\left(r_{2}\right) & M_{85}\left(r_{2}\right) & M_{86}\left(r_{2}\right) \\
M_{91}\left(r_{2}\right) & M_{92}\left(r_{2}\right) & M_{93}\left(r_{2}\right) & M_{94}\left(r_{2}\right) & 0 & 0
\end{array}\right|, \\
& \Delta_{22}=\left|\begin{array}{cccccc}
M_{11}\left(r_{1}\right) & M_{12}\left(r_{1}\right) & M_{13}\left(r_{1}\right) & M_{14}\left(r_{1}\right) & M_{15}\left(r_{1}\right) & M_{16}\left(r_{1}\right) \\
M_{21}\left(r_{1}\right) & M_{22}\left(r_{1}\right) & M_{23}\left(r_{1}\right) & M_{24}\left(r_{1}\right) & M_{25}\left(r_{1}\right) & M_{26}\left(r_{1}\right) \\
M_{51}\left(r_{1}\right) & M_{52}\left(r_{1}\right) & M_{53}\left(r_{1}\right) & M_{54}\left(r_{1}\right) & 0 & 0 \\
M_{71}\left(r_{2}\right) & M_{72}\left(r_{2}\right) & M_{73}\left(r_{2}\right) & M_{74}\left(r_{2}\right) & M_{75}\left(r_{2}\right) & M_{76}\left(r_{2}\right) \\
M_{81}\left(r_{2}\right) & M_{82}\left(r_{2}\right) & M_{83}\left(r_{2}\right) & M_{84}\left(r_{2}\right) & M_{85}\left(r_{2}\right) & M_{86}\left(r_{2}\right) \\
M_{91}\left(r_{2}\right) & M_{92}\left(r_{2}\right) & M_{93}\left(r_{2}\right) & M_{94}\left(r_{2}\right) & 0 & 0
\end{array}\right|
\end{aligned}
$$

where the elements $M_{j k}(r)$ are defined in the appendix [see Eq.(A.1)]. If the determinant $\left|\Delta_{22}\right|$ is singular, then it represents the frequency equation of guided circumferential waves in the hollow poroelastic cylinder for a permeable surface. This equation is similar (not same) to the frequency equation of plane-strain vibrations studied by Malla Reddy and Tajuddin [7] for a permeable surface. Then Eq.(4.13) reduces to $\left|\Delta_{12}\right|=0$ which represents the frequency equation of guided circumferential waves in a poroelastic solid cylinder of radius $r_{l}$ for a permeable surface. Similarly, the frequency equation of an impermeable surface (4.4) can be simplified in the form

$$
\left|\Delta_{2}\right|=\left|\begin{array}{cc}
\Delta_{11}^{*} & \Delta_{12}^{*} \\
\Delta_{21}^{*} & \Delta_{22}^{*}
\end{array}\right|=0
$$

where the sub-determinants $\Delta_{j k}^{*}(j, k=1,2)$ are exactly same as the sub-determinants defined in Eqs (4.14) with $M_{j k}(r)$ replaced by $N_{j k}(r)$, where $N_{j k}(r)$ are defined in the appendix [see Eq.(A.2)]. Analysis of Eq.(4.15), that is the frequency equation of an impermeable surface, is the same as that of the analysis of Eq.(4.13) described above.

\section{Non-dimensionalisation of frequency equation}

The wave number is real for propagating modes in a non-dissipative medium. The non-dimensional frequency $\Omega\left(\omega h / C_{0}{ }^{*}\right)$ as a function of the non-dimensional angular wave number is computed for two types of shaft-bearing models and the cylinder-piston models. The geometric parameter for the model under consideration is $g=r_{2} / r_{1}$. To analyze the frequency equations of permeable and impermeable surfaces, it is convenient to introduce the following non-dimensional parameters 


$$
\begin{aligned}
& a_{1}=P H^{*-1}, \quad a_{2}=Q H^{*-1}, \quad a_{3}=R H^{*-1}, \quad a_{4}=N H^{*-1}, \\
& m_{11}=\rho_{11} \rho^{*-1}, \quad m_{12}=\rho_{12} \rho^{*-1}, \quad m_{22}=\rho_{22} \rho^{*-1}, \\
& b_{1}=P^{*} H^{*-1}, \quad b_{2}=Q^{*} H^{*-1}, \quad b_{3}=R^{*} H^{*-1}, \quad b_{4}=N^{*} H^{*-1}, \\
& n_{11}=\rho_{11}^{*} \rho^{*-1}, \quad n_{12}=\rho_{12}^{*} \rho^{*-1}, \quad n_{22}=\rho_{22}^{*} \rho^{*-1}, \\
& x_{1}=\left(V_{0}^{*} V_{1}^{-1}\right)^{2}, \quad y_{1}=\left(V_{0}^{*} V_{2}^{-1}\right)^{2}, \quad z_{1}=\left(V_{0}^{*} V_{3}^{-1}\right)^{2}, \quad x_{1}^{*}=\left(V_{0}^{*} V_{1}^{*-1}\right)^{2}, \\
& y_{1}^{*}=\left(V_{0}^{*} V_{2}^{*-1}\right)^{2}, \quad z_{1}^{*}=\left(V_{0}^{*} V_{3}^{*-1}\right)^{2}
\end{aligned}
$$

where $H^{*}=P^{*}+2 Q^{*}+R^{*}, \quad \rho^{*}=\rho^{*}{ }_{11}+2 \rho^{*}{ }_{12}+\rho^{*}{ }_{22}$. Also $C_{0}^{*}$ and $V_{0}^{*}$ are reference velocities $\left(C^{*}{ }^{2}=N^{*} / \rho^{*}\right.$, $\left.V_{0}^{*}=H^{*} / \rho^{*}\right)$. Frequency Eqs (4.3), (4.4), (4.11) and (4.12) are non-dimensionalised employing Eq.(5.1). These equations constitute a relation between the non-dimensional frequency and angular wave number.

Parameters of two types of shaft-bearing assembly models are considered designated as Model-I and Model-II to compute the non-dimensional frequency. Model-I consists of a shaft made of sandstone saturated with kerosene (Fatt [13]) and the bearing made of sandstone saturated with water (Yew and Jogi [14]). Model-II consists of a shaft made of water saturated sandstone and a bearing made of kerosene saturated sandstone.

The non-dimensional physical parameters of Model-I and Model-II are given in Tab.1

\begin{tabular}{|c|c|c|c|c|c|c|c|c|c|c|}
\hline $\begin{array}{c}\text { Material } \\
\text { Parameter }\end{array}$ & $a_{1}$ & $a_{2}$ & $a_{3}$ & $a_{4}$ & $m_{11}$ & $m_{12}$ & $m_{22}$ & $x_{1}$ & $y_{1}$ & $z_{1}$ \\
\hline Model-I & 0.445 & 0.034 & 0.015 & 0.123 & 0.887 & -0.001 & 0.099 & 1.863 & 8.884 & 7.183 \\
\hline Model-II & 1.819 & 0.011 & 0.054 & 0.780 & 0.891 & 0 & 0.125 & 0.489 & 2.330 & 1.142 \\
\hline
\end{tabular}

\begin{tabular}{|c|c|c|c|c|c|c|c|c|c|}
\hline$b_{1}$ & $b_{2}$ & $b_{3}$ & $b_{4}$ & $n_{11}$ & $n_{12}$ & $n_{22}$ & $x_{1}{ }^{*}$ & $y_{1}{ }^{*}$ & $z_{1}{ }^{*}$ \\
\hline 0.960 & 0.006 & 0.028 & 0.412 & 0.877 & 0 & 0.123 & 0.913 & 4.347 & 2.129 \\
\hline 0.843 & 0.065 & 0.028 & 0.234 & 0.901 & -0.001 & 0.101 & 0.999 & 4.763 & 3.851 \\
\hline
\end{tabular}

\section{Numerical results and discussion}

Since stress wave propagation is the phenomenon of energy transfer, it plays a major role in fretting. Thus for the analysis of frequency equations of guided circumferential waves, Eqs (4.3), (4.4), (4.11) and (4.12) are non-dimensionalised employing Eqs (5.1) for computational work. For a given poroelastic model, these frequency equations constitute a relation between the non-dimensional frequency and angular wave 
number for fixed values of $g$. Different values of $g$, viz., 1.2362 and 5.0 are taken for numerical computation. The non-dimensional frequency is determined for different values of the angular wave number $\mathrm{n}$ and for fixed values of $g$, each for a permeable and an impermeable surface. The frequency of guided circumferential waves of first three modes of the shaft-bearing assembly for Model-I is presented in Fig.2 for a permeable and an impermeable surface. From the figure we see that waves are more dispersive in a permeable surface. The first two modes of an impermeable surface are almost linear. By increasing the thickness of the bearing, the frequency is obtained for Model-I and it is presented in Fig.3. Here we see that all the modes are linear when the wave number is more than 6 , and before it they are more dispersive. The frequency of an impermeable surface is less than that of a permeable surface for first three modes. Also, in general, it is seen that the frequency is larger for Model-I with an increased thickness. Figures 4 and 5 show the frequency as a function of the wave number for Model-II for the shaft-bearing assembly. The variation of frequency in Figure. 4 is similar to that in Fig.2 and in Fig.5 to that in Fig.3. The frequency of an impermeable surface is less than that of a permeable surface in this case also. The frequency is larger for Model-II than that of Model-I. The frequency of bore-piston assembly for Model-I is presented in Fig. 6 for a permeable and an impermeable surface. From the figure it is seen that the frequency of an impermeable surface is more that of a permeable surface when the wave number is more than 6 . And in this range, the frequency of a permeable surface is linear. This is in contrast with the shaft-bearing assembly. This is not the case for Model-II of the cylinder-piston assembly shown in Fig.7. Also, we see that the first mode of an impermeable surface is linear but not the other modes. Also, in general, the frequency for Model-II is more than that of Model-I. Also the frequency is obtained for the said Model-I and Model-II for a permeable and an impermeable surface for the shaft-bearing assembly and the cylinder-piston assembly for a small wave number in the interval $[0,1]$ and it is seen that the variation of frequency is similar as mentioned above.

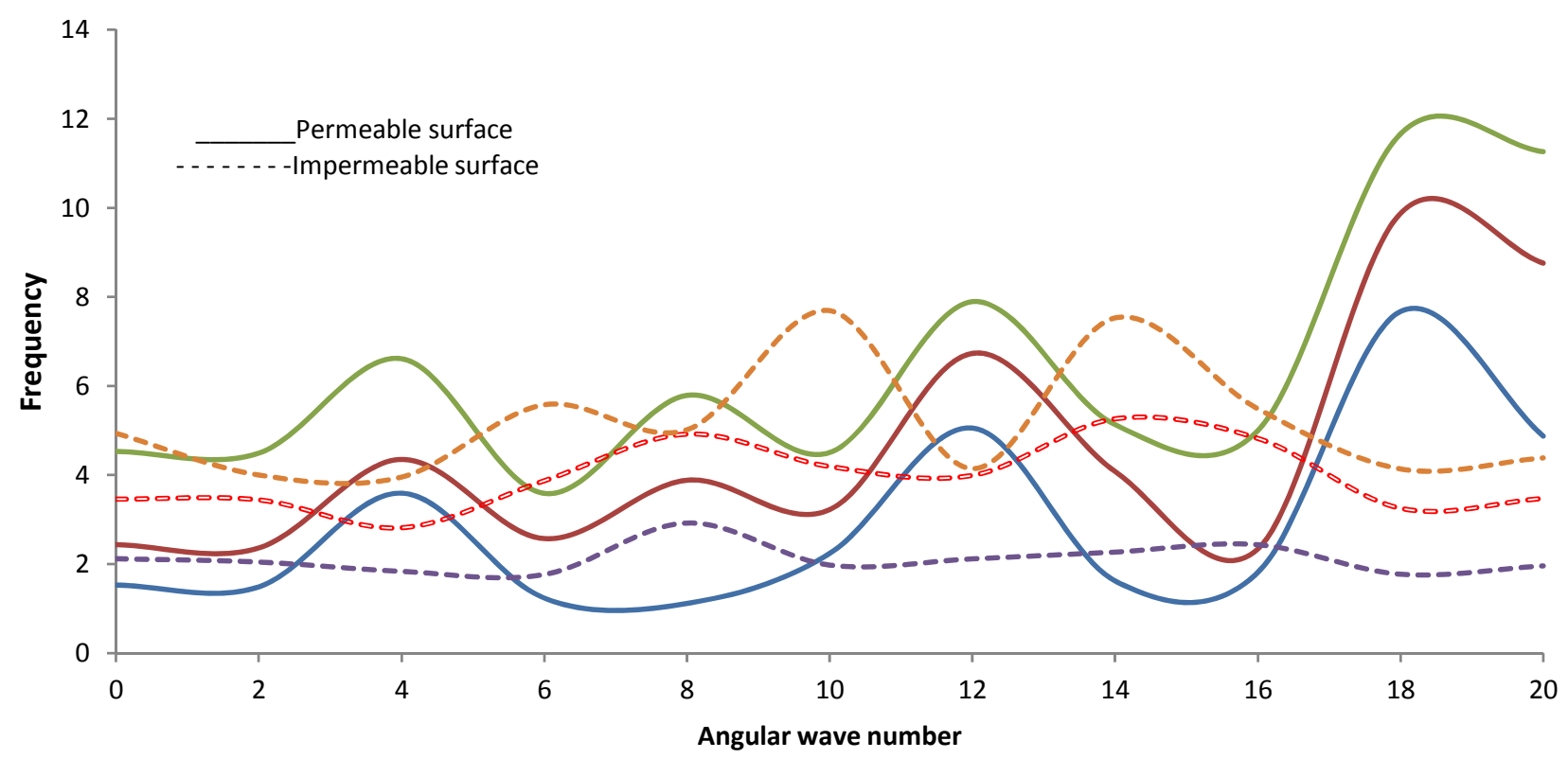

Fig.2. Frequency as a function of wave number (shaft-bearing assembly, Model-I, $g=1.2362$ ). 


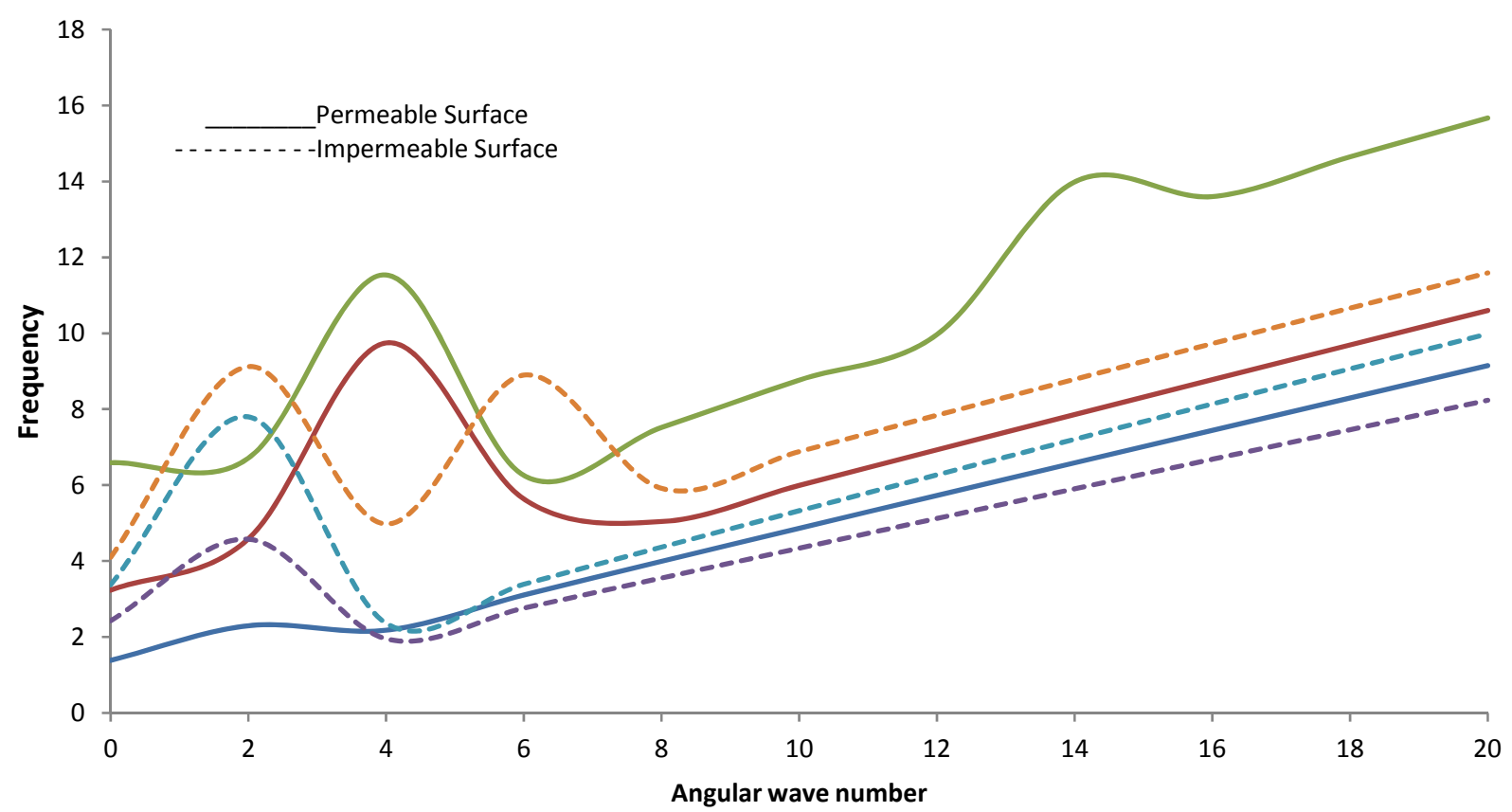

Fig.3. Frequency as a function of wave number (shaft-bearing assembly, Model-I, $g=5$ ).

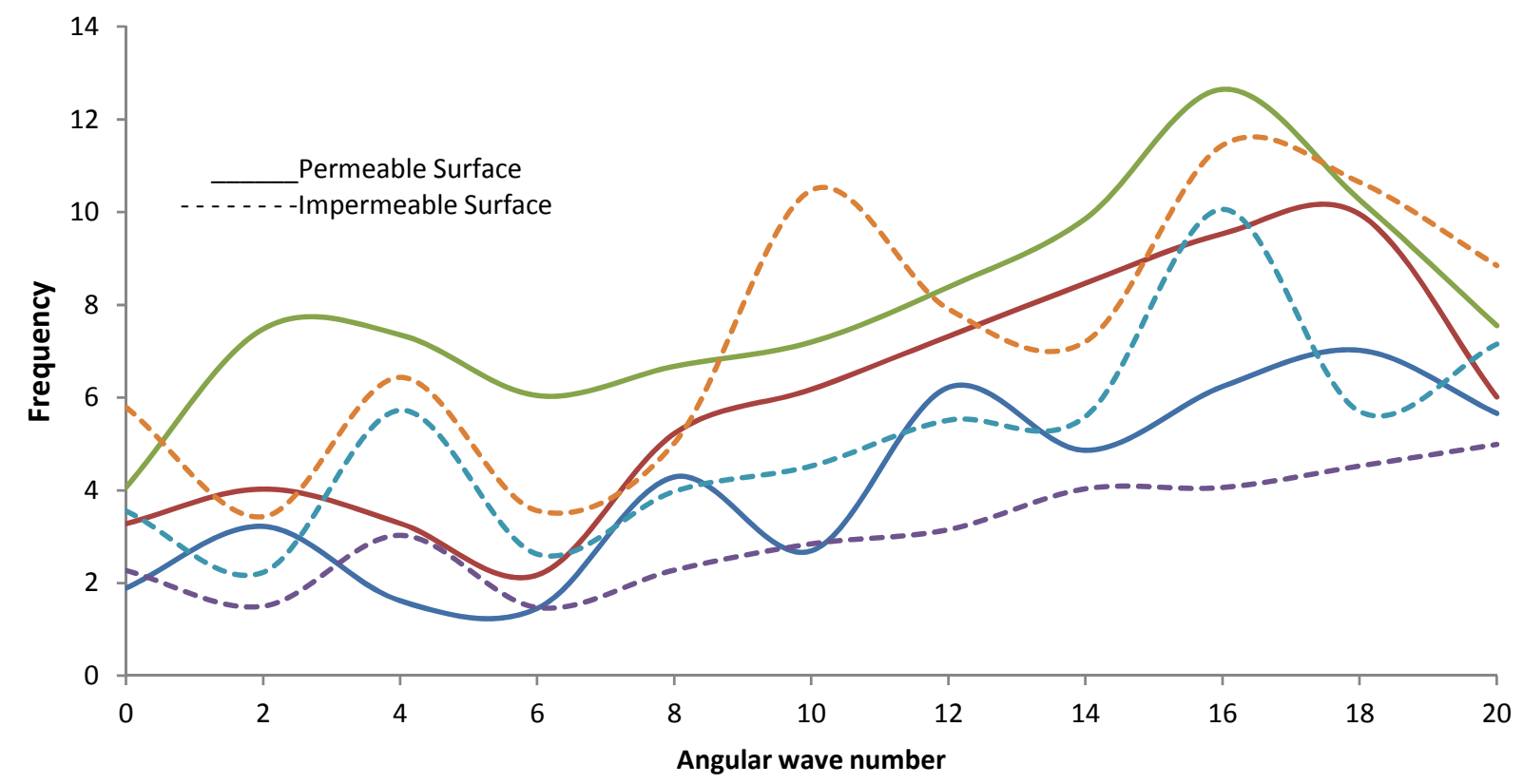

Fig.4. Frequency as a function of wave number (shaft-bearing assembly, Model-II, $g=1.2362$ ). 


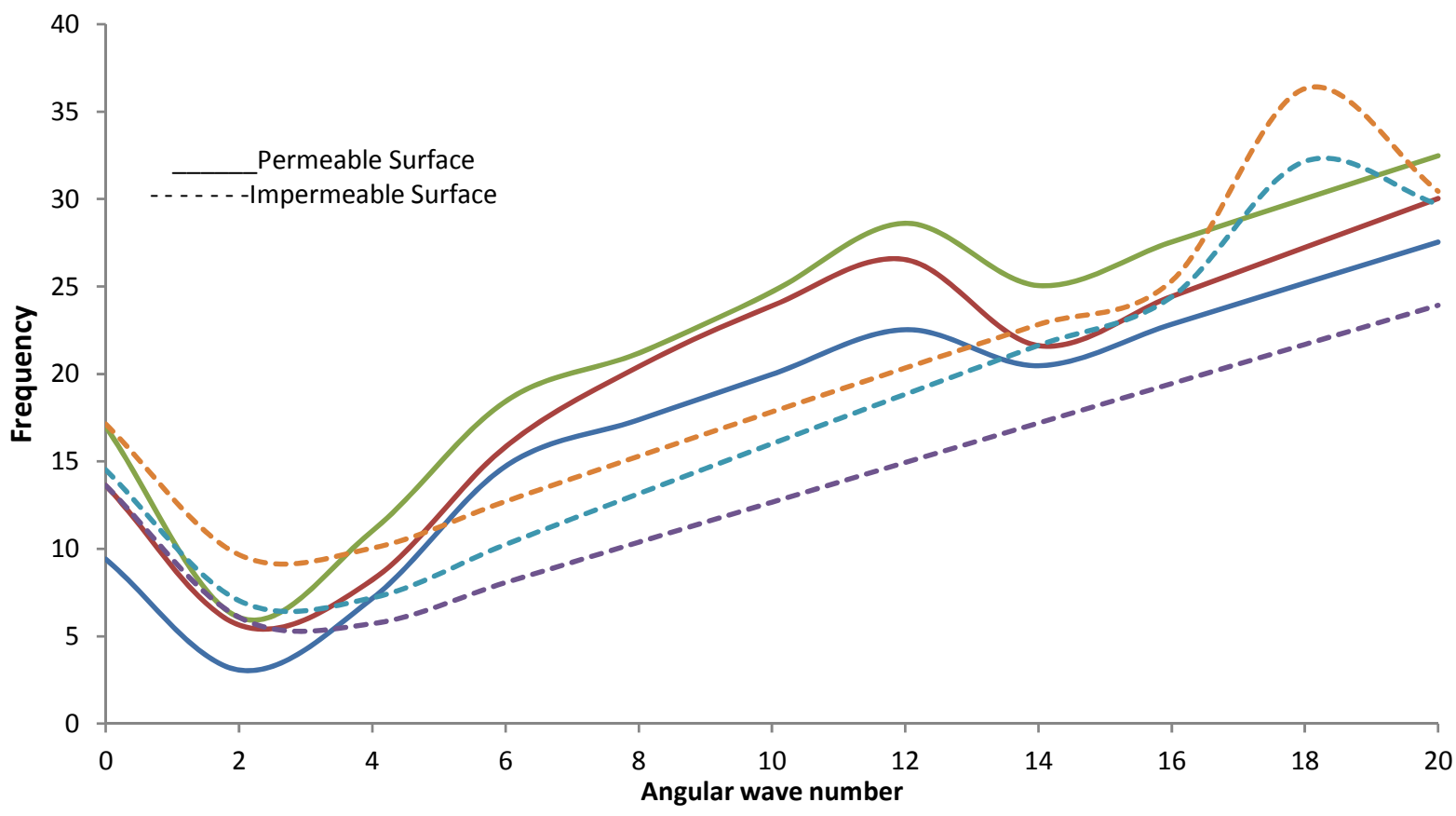

Fig.5. Frequency as a function of wave number (shaft-bearing assembly, Model-II, $g=5$ ).

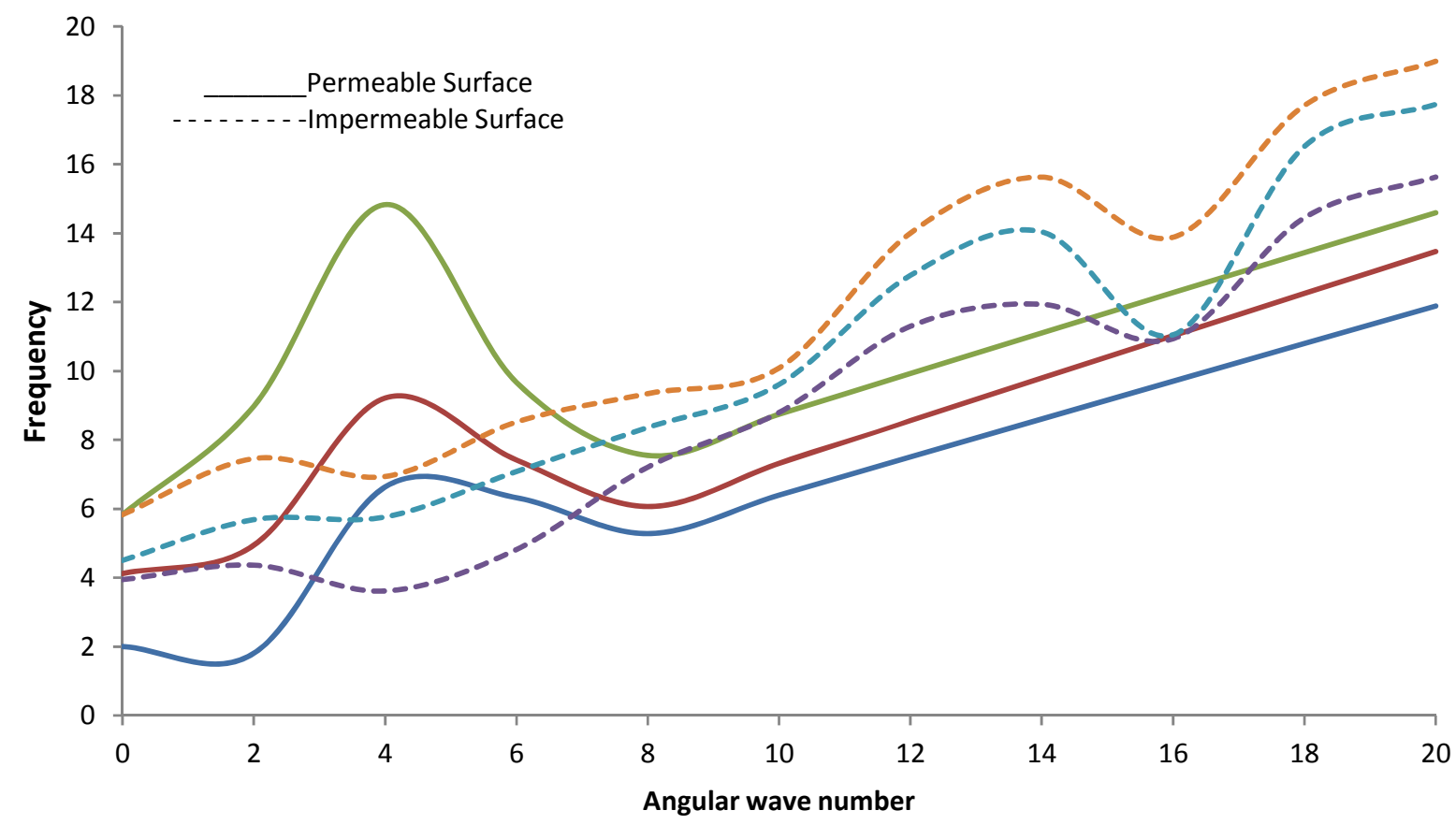

Fig.6. Frequency as a function of wave number (bore-piston assembly, Model-I). 


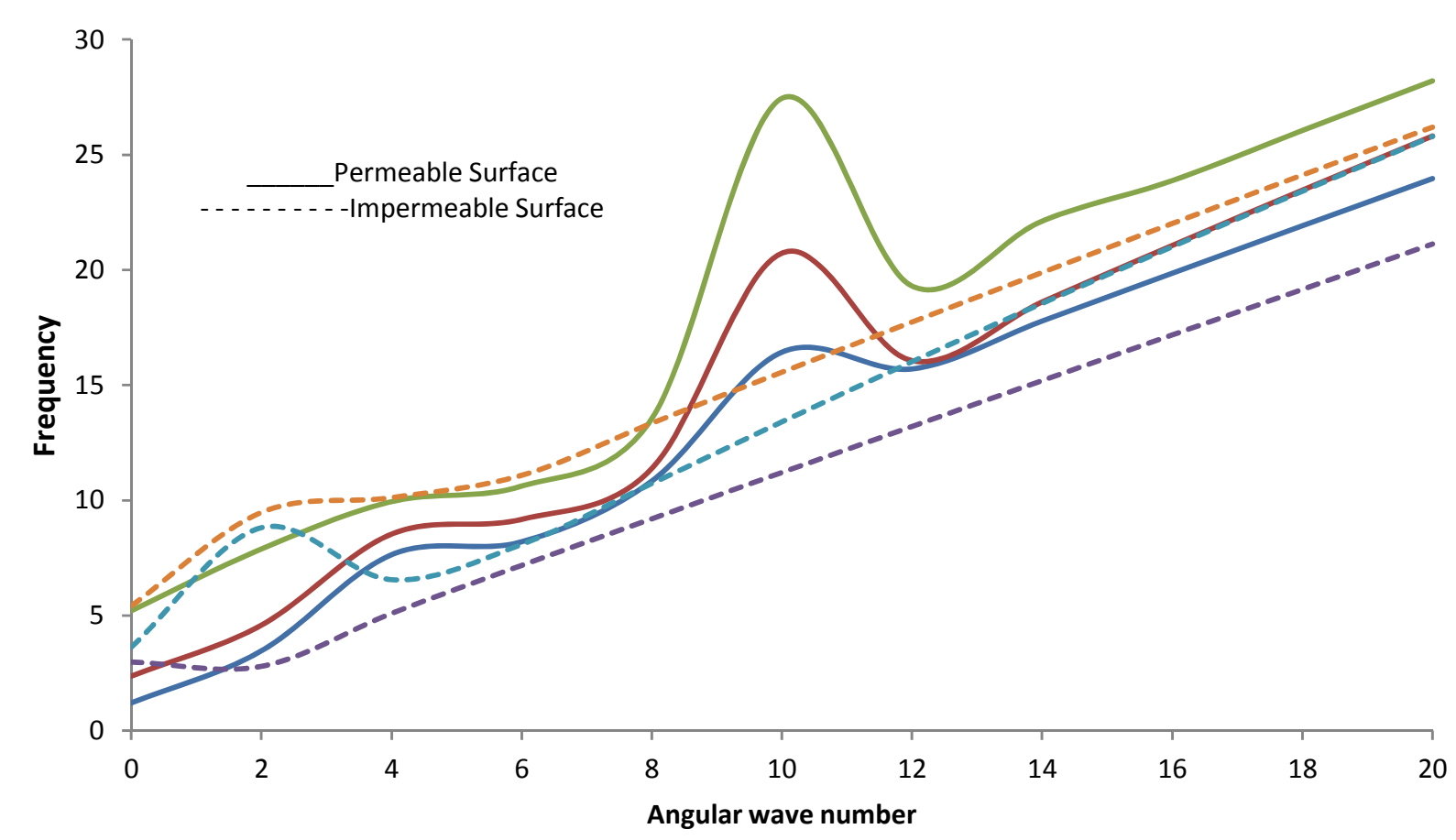

Fig.7. Frequency as a function of wave number (bore-piston assembly, Model-II).

\section{Appendix}

$$
\begin{aligned}
& M_{11}\left(r_{1}\right)=-\frac{2 N \xi_{1}}{r_{1}} J_{n}^{\prime}\left(\xi_{1} r_{1}\right)+\left\{2 N\left(\frac{n^{2}}{r_{1}^{2}}-\xi_{1}^{2}\right)+\left[(Q+R) \delta_{1}^{2}-(A+Q)\right] \xi_{1}^{2}\right\} J_{n}\left(\xi_{1} r_{1}\right), \\
& M_{12}\left(r_{1}\right)=-\frac{2 N \xi_{1}}{r_{1}} Y_{n}^{\prime}\left(\xi_{1} r_{l}\right)+\left\{2 N\left(\frac{n^{2}}{r_{1}^{2}}-\xi_{1}^{2}\right)+\left[(Q+R) \delta_{l}^{2}-(A+Q)\right] \xi_{1}^{2}\right\} Y_{n}\left(\xi_{1} r_{l}\right), \\
& M_{13}\left(r_{1}\right)=-\frac{2 N \xi_{2}}{r_{1}} J_{n}^{\prime}\left(\xi_{2} r_{1}\right)+\left\{2 N\left(\frac{n^{2}}{r_{1}^{2}}-\xi_{2}^{2}\right)+\left[(Q+R) \delta_{2}^{2}-(A+Q)\right] \xi_{2}^{2}\right\} J_{n}\left(\xi_{2} r_{1}\right), \\
& M_{14}\left(r_{1}\right)=-\frac{2 N \xi_{2}}{r_{1}} Y_{n}^{\prime}\left(\xi_{2} r_{1}\right)+\left\{2 N\left(\frac{n^{2}}{r_{1}^{2}}-\xi_{2}^{2}\right)+\left[(Q+R) \delta_{2}^{2}-(A+Q)\right] \xi_{2}^{2}\right\} Y_{n}\left(\xi_{2} r_{l}\right), \\
& M_{15}\left(r_{l}\right)=-\frac{2 N i n \xi_{3}}{r_{1}} J_{n}^{\prime}\left(\xi_{3} r_{l}\right)+\frac{2 N i n}{r_{l}^{2}} J_{n}\left(\xi_{3} r_{1}\right), \quad M_{16}\left(r_{1}\right)=-\frac{2 N i n \xi_{3}}{r_{l}} Y_{n}^{\prime}\left(\xi_{3} r_{l}\right)+\frac{2 N i n}{r_{l}^{2}} Y_{n}\left(\xi_{3} r_{l}\right) \\
& M_{17}\left(r_{l}\right)=\frac{2 N^{*} \xi_{1}^{*}}{r_{1}} J_{n}^{\prime}\left(\xi_{1}^{*} r_{1}\right)-\left\{2 N^{*}\left(\frac{n^{2}}{r_{1}^{2}}-\xi_{1}^{* 2}\right)+\left[\left(Q^{*}+R^{*}\right) \delta_{l}^{* 2}-\left(A^{*}+Q^{*}\right)\right] \xi_{1}^{* 2}\right\} J_{n}\left(\xi_{1}^{*} r_{l}\right),
\end{aligned}
$$




$$
\begin{aligned}
& M_{18}\left(r_{1}\right)=\frac{2 N^{*} \xi_{2}^{*}}{r_{1}} J_{n}^{\prime}\left(\xi_{2}^{*} r_{1}\right)-\left\{2 N^{*}\left(\frac{n^{2}}{r_{1}^{2}}-\xi_{2}^{* 2}\right)+\left[\left(Q^{*}+R^{*}\right) \delta_{2}^{* 2}-\left(A^{*}+Q^{*}\right)\right] \xi_{2}^{* 2}\right\} J_{n}\left(\xi_{2}^{*} r_{l}\right) \\
& M_{19}\left(r_{1}\right)=\frac{2 N^{*} i n \xi_{3}^{*}}{r_{1}} J_{n}^{\prime}\left(\xi_{3}^{*} r_{l}\right)-\frac{2 N^{*} i n}{r_{l}^{2}} J_{n}\left(\xi_{3}^{*} r_{1}\right), \quad M_{21}\left(r_{1}\right)=-\frac{2 N i n \xi_{1}}{r_{l}} J_{n}^{\prime}\left(\xi_{1} r_{l}\right)-\frac{N i n}{r_{l}^{2}} J_{n}\left(\xi_{1} r_{1}\right), \\
& M_{22}\left(r_{1}\right)=-\frac{2 N i n \xi_{1}}{r_{1}} Y_{n}^{\prime}\left(\xi_{1} r_{l}\right)-\frac{N i n}{r_{1}^{2}} Y_{n}\left(\xi_{1} r_{l}\right), \quad M_{23}\left(r_{1}\right)=-\frac{2 N i n \xi_{2}}{r_{1}} J_{n}^{\prime}\left(\xi_{2} r_{l}\right)-\frac{N i n}{r_{l}^{2}} J_{n}\left(\xi_{2} r_{1}\right), \\
& M_{24}\left(r_{1}\right)=-\frac{2 N i n \xi_{2}}{r_{1}} Y_{n}^{\prime}\left(\xi_{2} r_{l}\right)-\frac{N i n}{r_{l}^{2}} Y_{n}\left(\xi_{2} r_{l}\right), \quad M_{25}\left(r_{l}\right)=\frac{\xi_{3}}{r_{l}} J_{n}^{\prime}\left(\xi_{3} r_{l}\right)+\left(\xi_{3}^{2}-\frac{2 n^{2}}{r_{l}^{2}}\right) J_{n}\left(\xi_{3} r_{l}\right), \\
& M_{26}\left(r_{1}\right)=\frac{\xi_{3}}{r_{1}} Y_{n}^{\prime}\left(\xi_{3} r_{1}\right)+\left(\xi_{3}^{2}-\frac{2 n^{2}}{r_{1}^{2}}\right) Y_{n}\left(\xi_{3} r_{1}\right) \\
& M_{27}\left(r_{l}\right)=0, \quad M_{28}\left(r_{l}\right)=0, \quad M_{29}\left(r_{1}\right)=0, \\
& M_{31}\left(r_{1}\right)=0, \quad M_{32}\left(r_{1}\right)=0, \quad M_{33}\left(r_{1}\right)=0, \quad M_{34}\left(r_{1}\right)=0, \quad M_{35}\left(r_{1}\right)=0, \quad M_{36}\left(r_{1}\right)=0, \\
& M_{37}\left(r_{1}\right)=-\frac{2 N^{*} i n \xi_{1}^{*}}{r_{1}} J_{n}^{\prime}\left(\xi_{1}^{*} r_{1}\right)-\frac{N^{*} i n}{r_{1}^{2}} J_{n}\left(\xi_{1}^{*} r_{1}\right), \quad M_{38}\left(r_{1}\right)=-\frac{2 N^{*} i n \xi_{2}^{*}}{r_{1}} J_{n}^{\prime}\left(\xi_{2}^{*} r_{1}\right)-\frac{N^{*} i n}{r_{1}^{2}} J_{n}\left(\xi_{2}^{*} r_{1}\right), \\
& M_{39}\left(r_{1}\right)=\frac{\xi_{3}^{*}}{r_{1}} J_{n}^{\prime}\left(\xi_{3}^{*} r_{1}\right)+\left(\xi_{3}^{* 2}-\frac{2 n^{2}}{r_{1}^{2}}\right) J_{n}\left(\xi_{3}^{*} r_{1}\right), \\
& M_{41}\left(r_{l}\right)=\xi_{1} J_{n}^{\prime}\left(\xi_{1} r_{l}\right), \quad M_{42}\left(r_{l}\right)=\xi_{1} Y_{n}^{\prime}\left(\xi_{1} r_{l}\right), \quad M_{43}\left(r_{1}\right)=\xi_{2} J_{n}^{\prime}\left(\xi_{2} r_{l}\right), \\
& M_{44}\left(r_{1}\right)=\xi_{2} Y_{n}^{\prime}\left(\xi_{2} r_{l}\right), \quad M_{45}\left(r_{l}\right)=\frac{i n}{r_{l}} J_{n}\left(\xi_{3} r_{l}\right), \quad M_{46}\left(r_{l}\right)=\frac{i n}{r_{l}} Y_{n}\left(\xi_{3} r_{l}\right), \\
& M_{47}\left(r_{1}\right)=-\xi_{1}^{*} J_{n}^{\prime}\left(\xi_{1}^{*} r_{1}\right), \quad M_{48}\left(r_{1}\right)=-\xi_{2}^{*} J_{n}^{\prime}\left(\xi_{2}^{*} r_{1}\right), \quad M_{49}\left(r_{1}\right)=-\frac{i n}{r_{1}} J_{n}\left(\xi_{3}^{*} r_{1}\right), \\
& M_{51}\left(r_{l}\right)=\left(R \delta_{1}^{2}-Q\right) \xi_{1}^{2} J_{n}\left(\xi_{1} r_{l}\right), \quad M_{52}\left(r_{l}\right)=\left(R \delta_{l}^{2}-Q\right) \xi_{I}^{2} Y_{n}\left(\xi_{1} r_{l}\right), \\
& M_{53}\left(r_{1}\right)=\left(R \delta_{2}^{2}-Q\right) \xi_{2}^{2} J_{n}\left(\xi_{2} r_{1}\right), \quad M_{54}\left(r_{1}\right)=\left(R \delta_{2}^{2}-Q\right) \xi_{2}^{2} Y_{n}\left(\xi_{2} r_{1}\right), \\
& M_{55}\left(r_{1}\right)=0, \quad M_{56}\left(r_{1}\right)=0, \quad M_{57}\left(r_{1}\right)=0, \quad M_{58}\left(r_{1}\right)=0, \quad M_{59}\left(r_{1}\right)=0 ，
\end{aligned}
$$


$M_{61}\left(r_{1}\right)=0, \quad M_{62}\left(r_{1}\right)=0, \quad M_{63}\left(r_{1}\right)=0, \quad M_{64}\left(r_{1}\right)=0, \quad M_{65}\left(r_{1}\right)=0, \quad M_{66}\left(r_{1}\right)=0$,

$$
M_{67}\left(r_{1}\right)=\left(R^{*} \delta_{1}^{* 2}-Q^{*}\right) \xi_{1}^{* 2} J_{n}\left(\xi_{1}^{*} r_{1}\right), \quad M_{68}\left(r_{1}\right)=\left(R^{*} \delta_{2}^{* 2}-Q^{*}\right) \xi_{2}^{* 2} J_{n}\left(\xi_{2}^{*} r_{1}\right), \quad M_{69}\left(r_{1}\right)=0
$$$$
M_{71}\left(r_{2}\right)=-\frac{2 N \xi_{1}}{r_{2}} J_{n}^{\prime}\left(\xi_{1} r_{2}\right)+\left\{2 N\left(\frac{n^{2}}{r_{2}^{2}}-\xi_{1}^{2}\right)+\left[(Q+R) \delta_{1}^{2}-(A+Q)\right] \xi_{1}^{2}\right\} J_{n}\left(\xi_{1} r_{2}\right)
$$$$
M_{72}\left(r_{2}\right)=-\frac{2 N \xi_{1}}{r_{2}} Y_{n}^{\prime}\left(\xi_{1} r_{2}\right)+\left\{2 N\left(\frac{n^{2}}{r_{2}^{2}}-\xi_{1}^{2}\right)+\left[(Q+R) \delta_{1}^{2}-(A+Q)\right] \xi_{1}^{2}\right\} Y_{n}\left(\xi_{1} r_{2}\right),
$$$$
M_{73}\left(r_{2}\right)=-\frac{2 N \xi_{2}}{r_{2}} J_{n}^{\prime}\left(\xi_{2} r_{2}\right)+\left\{2 N\left(\frac{n^{2}}{r_{2}^{2}}-\xi_{2}^{2}\right)+\left[(Q+R) \delta_{2}^{2}-(A+Q)\right] \xi_{2}^{2}\right\} J_{n}\left(\xi_{2} r_{2}\right),
$$$$
M_{74}\left(r_{2}\right)=-\frac{2 N \xi_{2}}{r_{2}} Y_{n}^{\prime}\left(\xi_{2} r_{2}\right)+\left\{2 N\left(\frac{n^{2}}{r_{2}^{2}}-\xi_{2}^{2}\right)+\left[(Q+R) \delta_{2}^{2}-(A+Q)\right] \xi_{2}^{2}\right\} Y_{n}\left(\xi_{2} r_{2}\right),
$$$$
M_{75}\left(r_{2}\right)=-\frac{2 N i n \xi_{3}}{r_{2}} J_{n}^{\prime}\left(\xi_{3} r_{2}\right)+\frac{2 N i n}{r_{2}^{2}} J_{n}\left(\xi_{3} r_{2}\right)
$$$$
M_{76}\left(r_{2}\right)=-\frac{2 N i n \xi_{3}}{r_{2}} Y_{n}^{\prime}\left(\xi_{3} r_{2}\right)+\frac{2 N i n}{r_{2}^{2}} Y_{n}\left(\xi_{3} r_{2}\right), \quad M_{77}\left(r_{2}\right)=0, \quad M_{78}\left(r_{2}\right)=0, \quad M_{79}\left(r_{2}\right)=0
$$$$
M_{81}\left(r_{2}\right)=-\frac{2 N i n \xi_{1}}{r_{2}} J_{n}^{\prime}\left(\xi_{1} r_{2}\right)-\frac{N i n}{r_{2}^{2}} J_{n}\left(\xi_{1} r_{2}\right) \quad M_{82}\left(r_{2}\right)=-\frac{2 N i n \xi_{1}}{r_{2}} Y_{n}^{\prime}\left(\xi_{1} r_{2}\right)-\frac{N i n}{r_{2}^{2}} Y_{n}\left(\xi_{1} r_{2}\right),
$$$$
M_{83}\left(r_{2}\right)=-\frac{2 N i n \xi_{2}}{r_{2}} J_{n}^{\prime}\left(\xi_{2} r_{2}\right)-\frac{N i n}{r_{2}^{2}} J_{n}\left(\xi_{2} r_{2}\right) \quad M_{84}\left(r_{2}\right)=-\frac{2 N i n \xi_{2}}{r_{2}} Y_{n}^{\prime}\left(\xi_{2} r_{2}\right)-\frac{N i n}{r_{2}^{2}} Y_{n}\left(\xi_{2} r_{2}\right)
$$$$
M_{85}\left(r_{2}\right)=\frac{\xi_{3}}{r_{2}} J_{n}^{\prime}\left(\xi_{2} r_{2}\right)+\left(\xi_{3}^{2}-\frac{2 n^{2}}{r_{2}^{2}}\right) J_{n}\left(\xi_{3} r_{2}\right) \quad M_{86}\left(r_{2}\right)=\frac{\xi_{3}}{r_{2}} Y_{n}^{\prime}\left(\xi_{3} r_{2}\right)+\left(\xi_{3}^{2}-\frac{2 n^{2}}{r_{2}^{2}}\right) Y_{n}\left(\xi_{3} r_{2}\right)
$$

$$
M_{87}\left(r_{2}\right)=0, \quad M_{88}\left(r_{2}\right)=0, \quad M_{89}\left(r_{2}\right)=0,
$$$$
M_{91}\left(r_{2}\right)=\left(R \delta_{1}^{2}-Q\right) \xi_{1}^{2} J_{n}\left(\xi_{1} r_{2}\right), \quad M_{92}\left(r_{2}\right)=\left(R \delta_{1}^{2}-Q\right) \xi_{1}^{2} Y_{n}\left(\xi_{1} r_{2}\right),
$$$$
M_{93}\left(r_{2}\right)=\left(R \delta_{2}^{2}-Q\right) \xi_{2}^{2} J_{n}\left(\xi_{2} r_{2}\right), \quad M_{94}\left(r_{2}\right)=\left(R \delta_{2}^{2}-Q\right) \xi_{2}^{2} Y_{n}\left(\xi_{2} r_{2}\right),
$$

$$
M_{95}\left(r_{2}\right)=0, \quad M_{96}\left(r_{2}\right)=0, \quad M_{97}\left(r_{2}\right)=0, \quad M_{98}\left(r_{2}\right)=0, \quad M_{99}\left(r_{2}\right)=0 .
$$




$$
\begin{aligned}
& N_{j k}(r)=M_{j k}(r), \quad j=1,2,3,4,7,8, \quad \text { and } \quad k=1,2,3,4,5,6,7,8,9, \\
& N_{51}\left(r_{1}\right)=\left(R \delta_{1}^{2}-Q\right) \xi_{1}^{3} J_{n}^{\prime}\left(\xi_{1} r_{1}\right), \quad N_{52}\left(r_{1}\right)=\left(R \delta_{1}^{2}-Q\right) \xi_{1}^{3} Y_{n}^{\prime}\left(\xi_{1} r_{1}\right), \\
& N_{53}\left(r_{1}\right)=\left(R \delta_{2}^{2}-Q\right) \xi_{2}^{3} J_{n}^{\prime}\left(\xi_{2} r_{1}\right), \quad N_{54}\left(r_{1}\right)=\left(R \delta_{2}^{2}-Q\right) \xi_{2}^{3} Y_{n}^{\prime}\left(\xi_{2} r_{1}\right), \\
& N_{55}\left(r_{1}\right)=0, \quad N_{56}\left(r_{1}\right)=0, \quad N_{57}\left(r_{1}\right)=0, \quad N_{58}\left(r_{1}\right)=0, \quad N_{59}\left(r_{1}\right)=0, \\
& N_{61}\left(r_{1}\right)=0, \quad N_{62}\left(r_{1}\right)=0, \quad N_{63}\left(r_{1}\right)=0, \quad N_{64}\left(r_{1}\right)=0, \quad N_{65}\left(r_{1}\right)=0, \quad N_{66}\left(r_{1}\right)=0, \\
& N_{67}\left(r_{1}\right)=\left(R^{*} \delta_{1}^{* 2}-Q^{*}\right) \xi_{1}^{* 3} J_{n}^{\prime}\left(\xi_{1}^{*} r_{1}\right), \quad N_{68}\left(r_{1}\right)=\left(R^{*} \delta_{2}^{* 2}-Q^{*}\right) \xi_{2}^{* 3} J_{n}^{\prime}\left(\xi_{2}^{*} r_{1}\right), \quad M_{69}\left(r_{1}\right)=0, \\
& N_{91}\left(r_{2}\right)=\left(R \delta_{1}^{2}-Q\right) \xi_{1}^{3} J_{n}^{\prime}\left(\xi_{1} r_{2}\right), \quad N_{92}\left(r_{2}\right)=\left(R \delta_{1}^{2}-Q\right) \xi_{1}^{3} Y_{n}^{\prime}\left(\xi_{1} r_{2}\right), \\
& N_{93}\left(r_{2}\right)=\left(R \delta_{2}^{2}-Q\right) \xi_{2}^{3} J_{n}^{\prime}\left(\xi_{2} r_{2}\right), \quad N_{94}\left(r_{2}\right)=\left(R \delta_{2}^{2}-Q\right) \xi_{2}^{3} Y_{n}^{\prime}\left(\xi_{2} r_{2}\right), \\
& N_{95}\left(r_{2}\right)=0, \quad N_{96}\left(r_{2}\right)=0, \quad N_{97}\left(r_{2}\right)=0, \quad N_{98}\left(r_{2}\right)=0, \quad N_{99}\left(r_{2}\right)=0 .
\end{aligned}
$$

\section{Nomenclature}

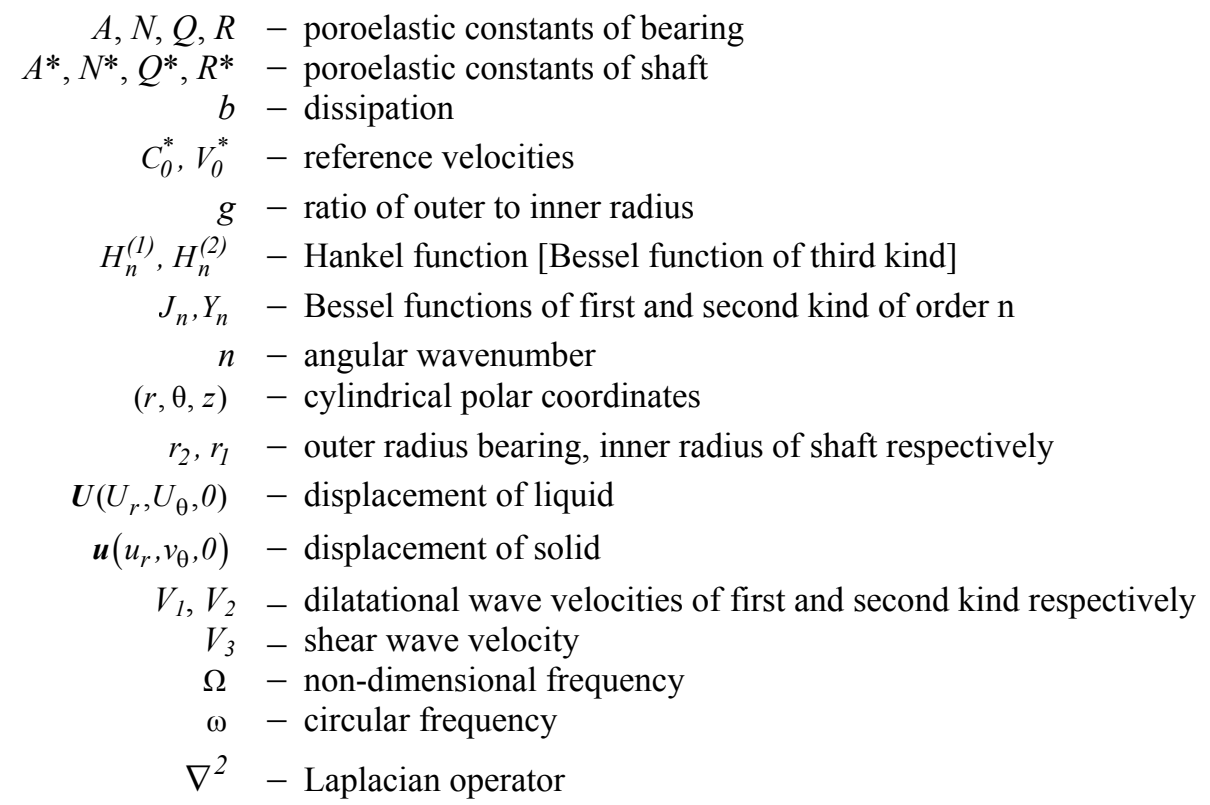

\section{References}

[1] Nagy P.B., Blodgett M. and Godis M. (1994): Weep hole inspection by circumferential creeping waves. - NDT and E., vol.27, pp.131-142.

[2] Christine Valle, Jianmin Qu and Jacobs L.J. (1999): Guided circumferential waves in layered cylinders. International Journal of Engineering Science, vol.37, pp.1369-1387. 
[3] Tajuddin M. and Ahmed Shah S. (2006): Circumferential waves of infinite hollow poroelastic cylinders. - Trans. ASME, J. Appl. Mech., vol.73, pp.705-708.

[4] Tajuddin M. and Ahmed Shah S. (2007): On torsional vibration of infinite hollow poroelastic cylinders. - Journal of Mechanics of Materials and Structures, vol.2, pp.189-200.

[5] Whittier J.S. and Jones J.P. (1967): Axially symmetric wave propagation in a two-layered cylinder. - International Journal of Solids and Structures, vol.3, pp.657-675.

[6] Liu G. and Qu J. (1998): Guided circumferential waves in a circular annulus. - Trans. ASME, J. Appl. Mech., vol.65, pp.424-430.

[7] Malla Reddy P. and Tajuddin M. (2000): Exact analysis of the plane-strain vibrations of thick-walled hollow poroelastic cylinders. - International Journal of Solids and Structures, vol.37, pp.3439-3456.

[8] Towfighi S. Kundu T. and Ehsani M. (2002): Elastic wave propagation in circumferential direction in anisotropic cylindrical curved plates. - Trans. ASME. J. Appl. Mechanics, vol.69, pp.283-291.

[9] Ahmed Shah S. (2011): Flexural wave propagation in coated poroelastic cylinders with reference to fretting fatigue. - Journal of Vibration and Control, vol.17, pp.1049-1064.

[10] Biot M.A. (1956): Theory of propagation of elastic waves in fluid-saturated porous solid. - J. Acoust. Soc. Am., vol.28, pp.168-178.

[11] Viktorov I.A. (1958): Rayleigh-type waves on curved surfaces. - J. Acoust. Soc. Am, vol.4, pp.131-136.

[12] Tajuddin M. and Sarma K.S. (1980): Torsional vibrations of poroelastic cylinders. - Trans. ASME, J. Appl. Mech., vol.47, pp.214-216.

[13] Fatt I. (1959): The Biot-Willis elastic coefficients for a sandstone. - J. Appl. Mech., vol.26, pp.296-297.

[14] Yew C.H. and Jogi P.N. (1976): Study of wave motions in fluid-saturated porous rocks. - J. Acoust. Soc. Am, vol.60, pp.2-8.

[15] Abramowitz A. and Stegun I.A. (1965): Handbook of Mathematical Functions. - National Bureau of Standards, Washington, D.C.

Received: August 15, 2015

Revised: April 12, 2016 